\title{
Study on the ATP-ase Activity of the Proximal Tubule Cell Membranes of the Rabbit Kidney
}

\author{
Kenzo Oshima, Michinobu Hatano, Nobuhiro Sugino, \\ Issei Kabeyazawa, Makio Takahashi, Hiroshi Yamamoto, \\ Motozo Hara, and Hiroya Sugeno \\ Nihon Univ. School of Medicine Prof. Oshima's clinic of Medicine.
}

\section{Introduction}

In an attempt to elucidate the relationship between the function and ultramicrostructure of the renal proximal tubule cells of the rabbit, histochemical staining for ATP-ase of the cell was performed under the normal and pathological conditions.

\section{Method}

Normal adult male rabbits, divided to the normal group (5 rabbits), eggalbumin loaded group (15 rabbits), and Masugi-nephritis group (5 rabbits), were objects of these experiments and the kidneys were examined by means of ATP-ase staining technic under the light and electron microscopic investigation.

Simultaneously the kidney tissue was homogenized and the cell fractions were isolated by the ultracentrifuge (Schneider's method).

The ATP-ase activity of each cell fraction was chemically determined in order to compare with the results obtained by histochemical staining procedure. The ATP-ase stainig for the light microscope was performed by means of lead phosphate method (Gomori and Wachstein), whereas that for the electron microscope was done by the Esner-Novikoff's method.

The ATP-ase activity was measured by the Iwasa, Imamoto and Okunuki's method, with varing the concentration of substrate, $\mathrm{pH}$, temperature and time.

The peroxidase staining was made by means of De Robertis Grasso's technic.

\section{Result}

As far as the normal group is concerned, light microscopic finding is not sufficient enough to detect what part of the cell has ATP-ase activity.

The figure 1 shows the light microscopic picture of the normal rabbit kidney. The dark-brown colored parts are showing positive ATP-ase reaction. From this picture, ATP-ase reaction appears to be markedly positive at the apical cell zone.

The figure 2 shows the electron microscopic picture. High ATP-ase activity is found at the site of the cell membrane, particularly brush border. 
Conversely ATP-ase activity is also positive at the cell membrane of the basal cell zone. (the figure 3 )

In general, pinocytosis appears to be quite active at the renal proximal tubule cell and also there is high ATP-ase activity at the apical cell zone just like intestine mucosa and liver cell which have also active pinocytosis. The high ATP-ase activity is found at the cell membrane and this enzyme seems to be important factor for the energy source of pinocytosis. In addition, this

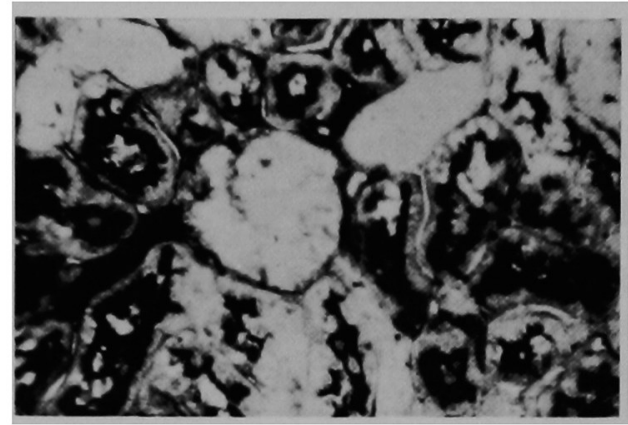

Fig. 1 High ATP-ase activity (By Wachstein's staining Method) at the brush border and granules of the proximal tubule of the rabbit kidney.
Fig. 2 ATP-ase activity on the electron microscopic picture. (brush border)

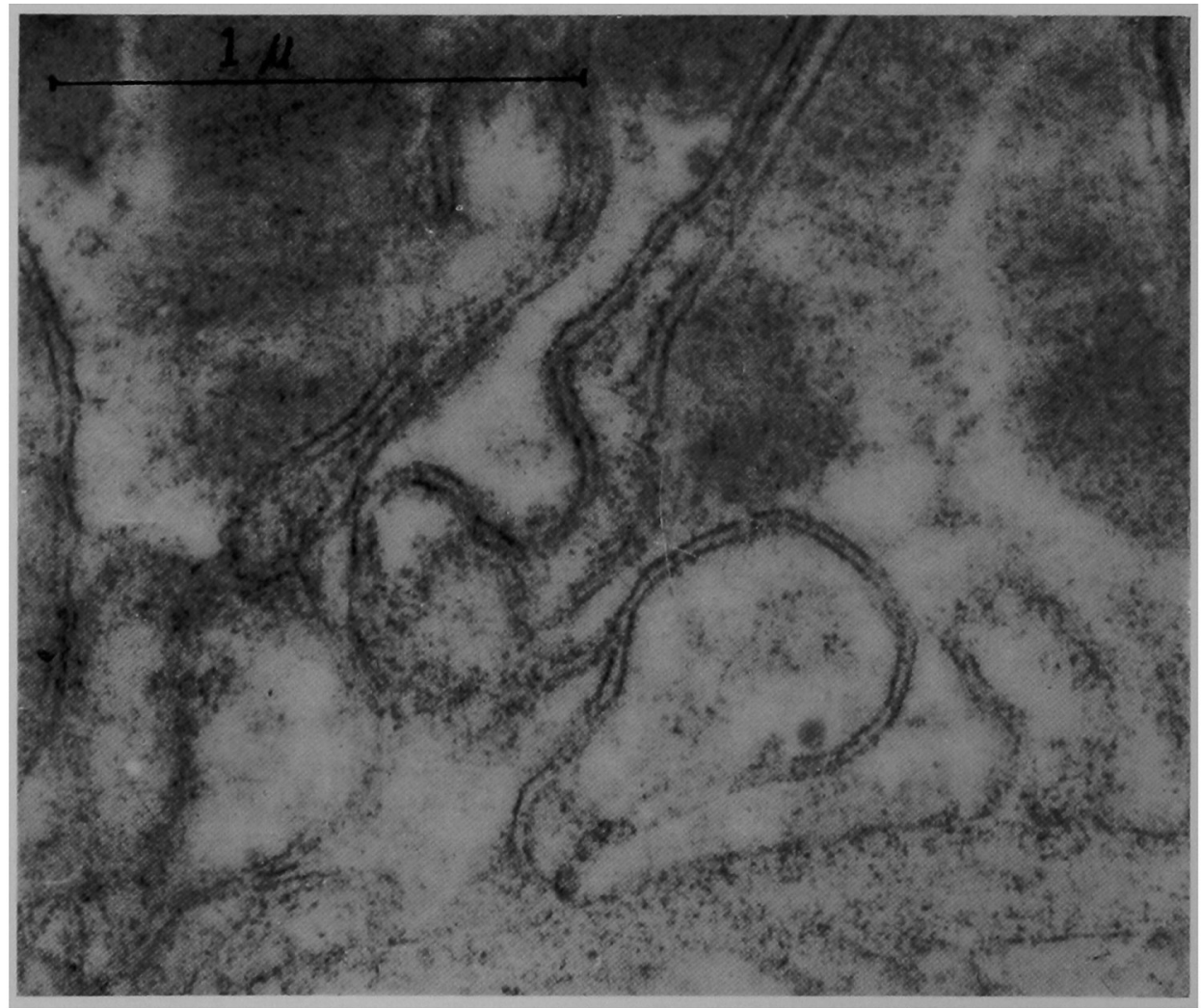

Fig. 3 ATP-ase activity on the electron microscopic picture. (basal cell zone) 
enzyme is apparently one of the factor of keeping elasticity of the cell membrane and maintaining brush border as usual.

From the point of view of protein absorption, egg albumin was injected to the rabbit and tissue materials were taken at 40 minutes, 3 hours and 12 hours following the injection respectively.

The figure 4 shows ATP-ase staining at 40 minutes following the injection of egg albumin and ATP-ase activity was increased.

The figure 5 is the tissue of 3 hours following the injection. ATP-ase activity was decreased. Therefore, microsome fraction is isolated from the homogenate of the kidney cortex by means of ultracentrifuge and biochmical determination of ATP-ase activity was done.

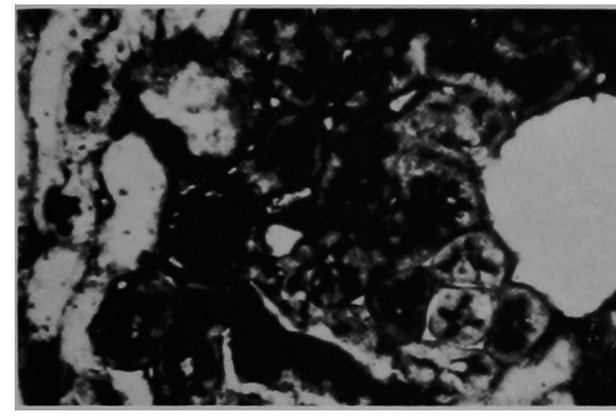

Fig. 4 ATP-ase staining at 40 minutes following the injection of egg-albumin.

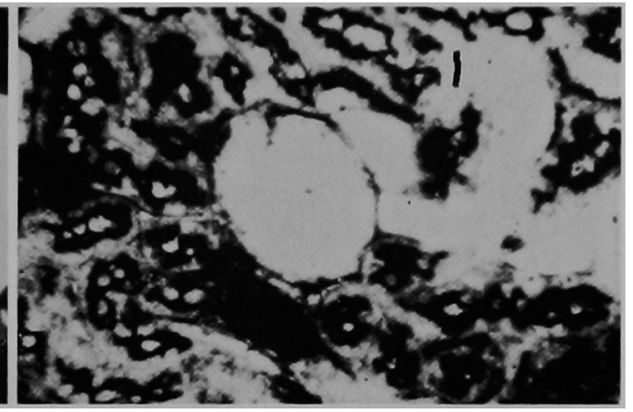

Fig. 5 ATP-ase staining at 3 hours following the injection of egg-albumin.

The figure 6 is showing high ATP-ase activity at 40 minutes, although there are low ATP-ase activities at 3 and 12 hours. These results from the chemical determination are in good agreement with the previous histochemical staining. The high ATP-ase activity is found during the active pinocytosis and after that pinocytosis become inactive and ATP-ase activity falls.

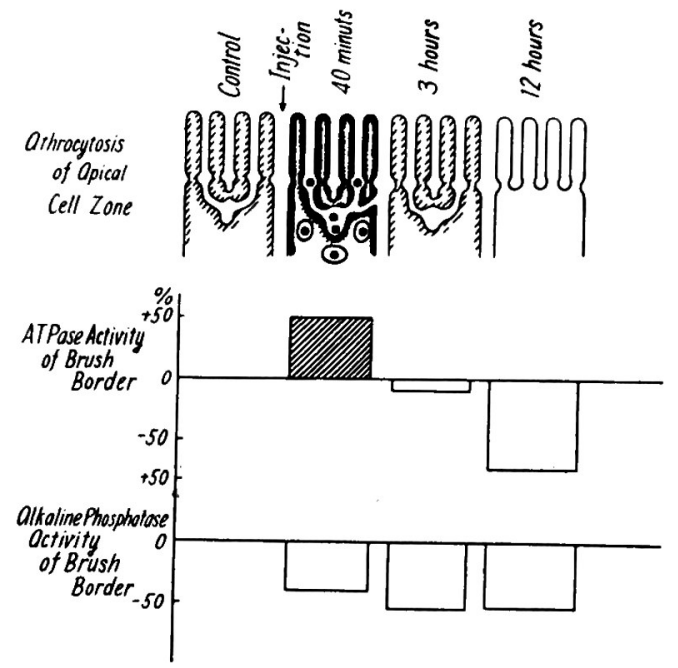

Fig. 6 Relationship between athrocytosis of brush border and ATP-ase activity of brush border. 
Last point is how pinocytosis does change when ATP-ase activity of the cell membrane decreases under the pathologic condition, such as Masugi nephritis.

From the PAS staining of Masugi nephritis (the figure 7), there is no definite degeneration in the proximal tubule cell as compared to the typical inflammation of glomeruli.

The figure 8 shows ATP-ase activity seems to disappear at the apical cell zone.

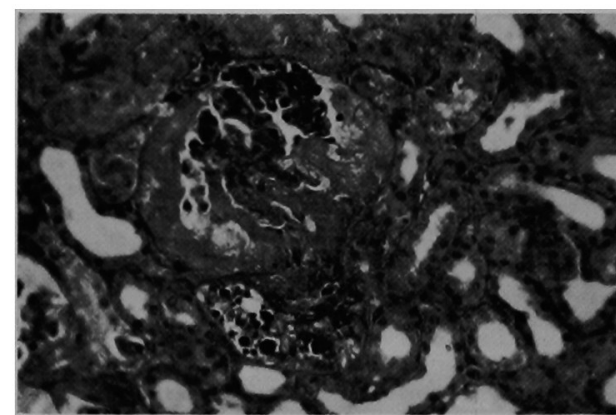

Fig. 7 PAS staining of Masugi nephritis.

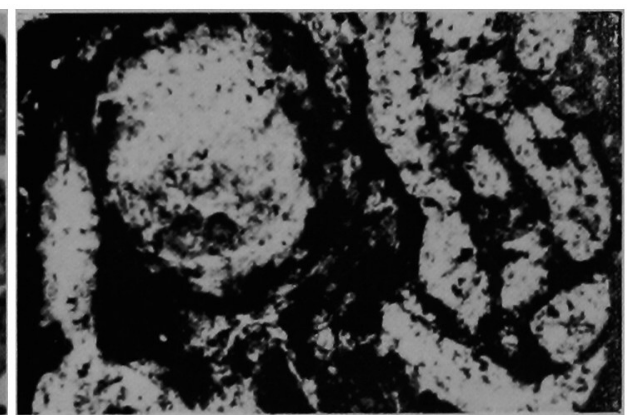

Fig. 8 Disappearance of ATP-ase activity at the apical cell zone.

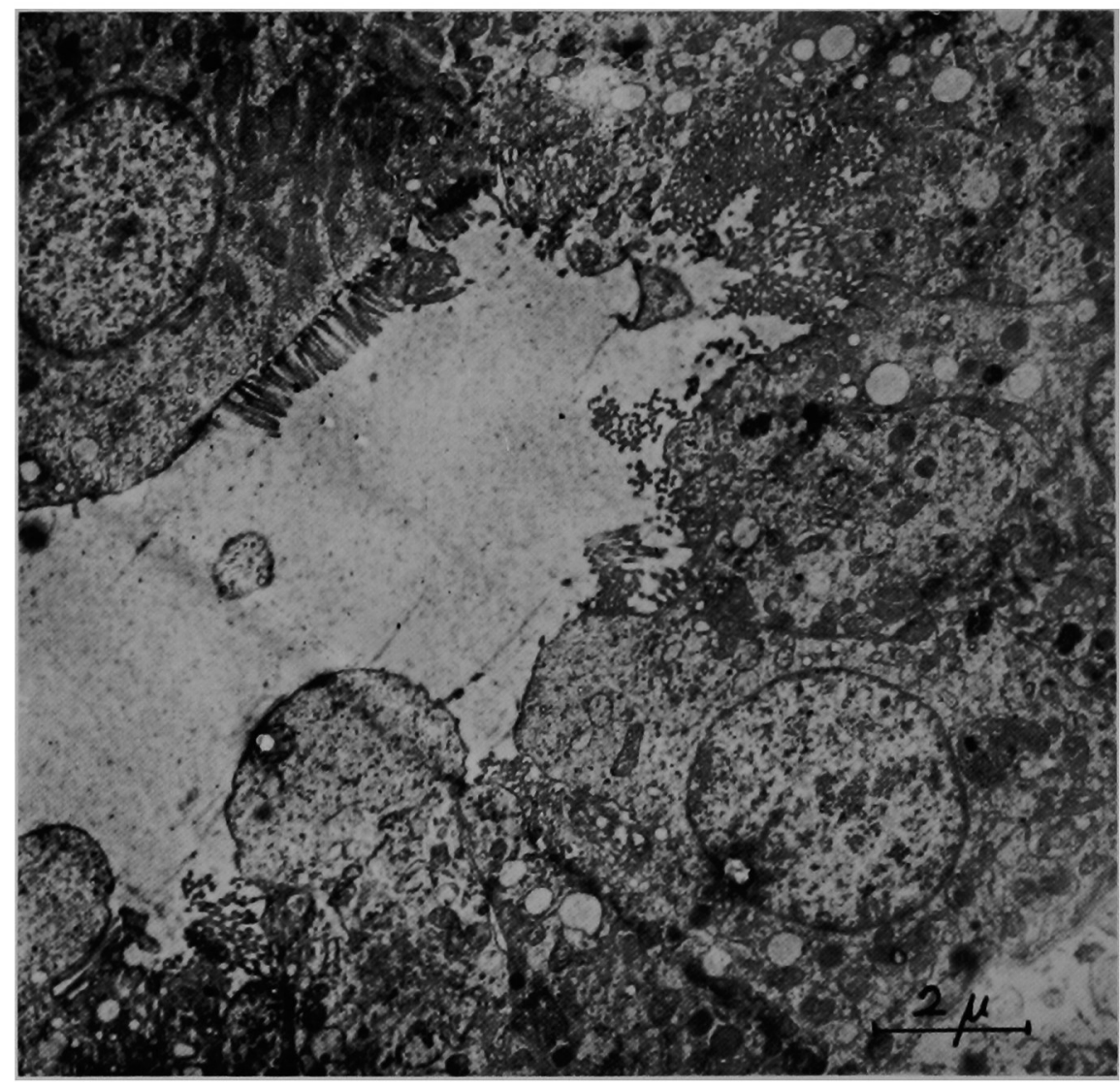

Fig. 9 Electron microscopic picture of Masugi nephritis. 
Electron microscope indicates disappearance and destruction of the brush border which implies that the decrease or loss of ATP-ase activity results in the destruction of the brush border. (the figure 9)

As the next experiment, we injected peroxidase, of which molecular weight is about 40,000 , instead of egg-albumin. Peroxidase is filtered by glomerular capillaries and absorbed by the proximal tubule cells, so that peroxidase staining of the kidney tissue shows this particular substance seems to be transported by the proximal tubule cells.

The figure 10 indicates peroxidase staining of the normal rabbit and blue granula are found in the proximal tubule cells.

However, as the figure 11 shows, in Masugi nephritis of which ATP-ase activity decreases, no peroxidase is taken up by the proximal tubule cells, although this substance is filtered by the glomerulus. There is definite decrease in pinocytotic activity when the ATP-ase activity of the cell membrane is lowered.

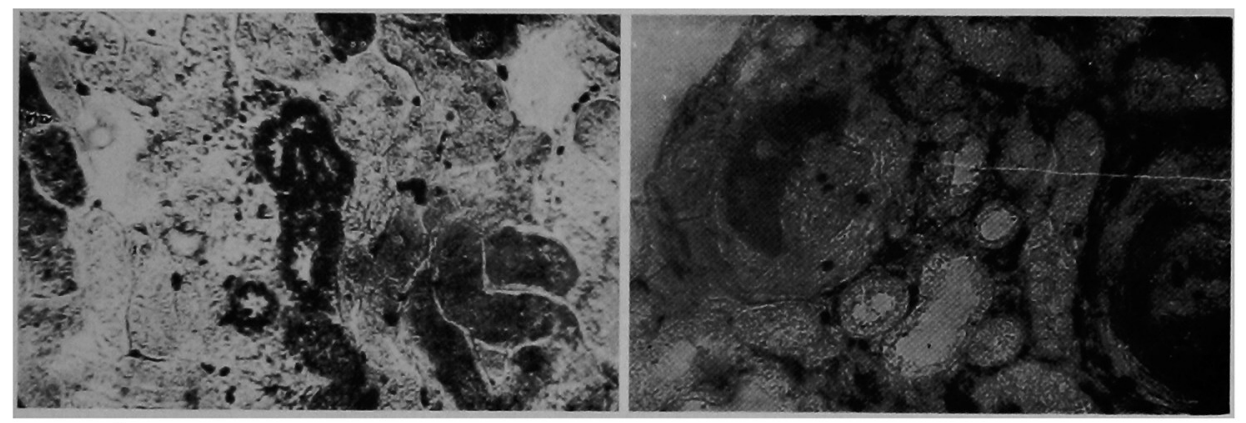

Fig. 10 Peroxidase staining of normal rabbit.

Fig. 11 Rabbit with the Masugi nephritis accompanied with the injection of peroxidase.

\section{Conclusion}

The ATP-ase activity is highly located at the cell membrane of the proximal tubule cell of the rabbit kidney and this enzyme appears to be important to maintain the normal structure of the brush border and also to maintain the elasticity of the cell membrane.

In addition, ATP-ase is also considered to play an important role in terms of cell membrane movement, including pinocytosis.

\section{Discussion}

Dr. Hashimoto :

1) By use of metal method, the precipitation of metals often may occur with different localization from that of enzyme activity, i. e. at cellular and nuclear membrane.

2) Are contained cellular membranes in the microsome fraction?

Dr. Ogawa: Plasma membrane surely has metalophilic tendendency to lead. We must observe the sufficient control sections immersed in substrate omitted solutions. In the present photographs, the reactions of brush border seemed to be excessive and to be contaminated with other nonspecific reactions.

Dr. Hatano: (To Dr. Hashimoto) In regard to metalophilia, we need further experiments, 
but it is surely observed that staining reactions coincide with the biochemical results of cellular fractions.

(To Dr. Ogawa) We showed the lineal staining of brush border like Essmer-Novikoff's findings.

\title{
Histochemical Studies on Copper Metabolism
}

\author{
Shigeo OKinaka, Masaki Yoshikawa, Toshiji MozaI, \\ Haruo Watanabe, Toshio Terao, Kazuaki Ogiwara \\ and Shunsaku HiraI.
}

The Third Department of Internal Medicine (Director: Prof. S. Okinaka, M. D.), Faculty of Medicine, University of Tokyo.

The mechanism of deposition of copper in the tissue of patients with hepatolenticular degeneration (HLD) is not fully understood.

We studied on copper metabolism in intra-uterine life, in patients with HLD as well as ordinary acquired liver diseases, and in experimental copper intoxication in relation to the pathogenesis of HLD.

\section{Material and Methods}

\section{Materials}

1. Copper and ceruloplasmin concentrations in the serum obtained from the patients with HLD, other acquired liver diseases and from the cubital vein and umbilical cord of normal healthy pregnant women at the time of delivery were estimated.

2. The livers of 8 cases of HLD, of 24 cases of other acquired liver diseases, and of 19 cases of fetuses, all fixed in $10^{\circ}$ neutralized formalin solution for quantitative and or histochemical analysis for copper.

3. The livers of copper intoxicated adult dogs were analysed for copper biochemically as well as histochemically.

Methods

Serum ceruloplasmin concentration was determined using Ravin's method ${ }^{12}$. Copper content in serum and tissue were determined using the method previously reported ${ }^{2}$. Histochemical demonstration for copper was performed according to Okamoto and Utamura ${ }^{8)}$. In addition, the livers of HLD were studied using the method of Fredericq and Schevremont" for the demonstration of reducing radical of protein.

\section{Results}

In our study, following points were demonstrated.

1. The concentration of serum ceruloplasmin in the blood from umbilical 